\title{
Human Eosinophils Express Functional Interleukin 2 Receptors
}

\author{
Thomas H. Rand," David S. Silberstein," Hardy Kornfeld," and Peter F. Weller \\ *Harvard Thorndike Laboratory and Charles A. Dana Research Institute, Department of Medicine, Beth Israel and \\ ${ }^{\ddagger}$ Brigham and Women's Hospitals, Harvard Medical School, Boston, Massachusetts 02215; and \\ \$Pulmonary Center, Boston University School of Medicine, Boston, Massachusetts 02118
}

\begin{abstract}
Because $T$ cell-derived cytokines may affect the functioning of eosinophils, we have investigated the capacity of human eosinophils to respond to IL-2. IL-2 was a potent chemoattractant with $\mathrm{ED}_{50}$ of $10^{-12} \mathrm{M}$ with eosinophils from all normal and eosinophilic donors tested. The monoclonal antibodies anti-Tac and TU27 against p55 (Tac/CD25) and p75 receptor subunits, respectively, each inhibited IL-2-dependent eosinophil migration. The molar potency of IL-2 and the inhibitory activity of each of the above antibodies suggest that high affinity heterodimeric IL-2 receptor complexes mediated the migration responses of eosinophils to IL-2. Binding of monoclonal antibody against p75 was not detectable by flow cytometry, and high affinity binding sites for ${ }^{125} \mathrm{I}-\mathrm{IL}-2$ were below the limits of quantitation on eosinophils from individuals with eosinophilia. Expression of p55 (Tac/CD25) by eosinophils, without requirement for in vitro activation, was demonstrable by flow cytometry, radioimmunoprecipitation, and Northern blotting for mRNA. Surface expression of p55 on eosinophils from normal or eosinophilic individuals increased during culture for 24-48 h with a biologic activity purified from stimulated U937 cells and to a lesser extent with granulocyte-macrophage CSF or lymphocyte chemoattractant factor but not with nine other cytokines. These studies indicate that blood eosinophils respond to IL-2 and identify one mechanism whereby activation of T lymphocytes may influence the function of eosinophils. (J. Clin. Invest. 1991. 88:825-832.) Key words: adult $T$ cell leukemiaderived factor • chemotaxis • eosinophil cytotoxicity-enhancing factor $\bullet$ granulocyte $\bullet$ lymphocyte chemoattractant factor
\end{abstract}

\section{Introduction}

Development of high affinity receptors for IL-2 is a crucial process in proliferative responses of lymphoid cells. The current model of the high affinity IL-2 receptor (IL-2R) complex includes, at a minimum, two noncovalently associated subunits, $\mathrm{p} 55$ and p75 (1). Individually, p55 and p75 bind IL-2 with low or intermediate affinity, respectively (2-4). The p55 subunit, designated CD25, is recognized by antibodies such as anti-Tac (5). In lymphocytes, p 55 is transcriptionally regulated; induction of this peptide correlates with acquisition of

Address correspondence and reprint requests to Dr. Peter F. Weller, Division of Infectious Diseases, Beth Israel Hospital, DA 617, 330 Brookline Avenue, Boston, MA 02215.

Received for publication 4 December 1990 and in revised form 19 March 1991.

J. Clin. Invest.

(c) The American Society for Clinical Investigation, Inc.

0021-9738/91/09/0825/08 $\$ 2.00$

Volume 88, September 1991, 825-832 high affinity binding sites and biologic responsiveness to IL-2 during lymphocyte activation (1).

In addition to the studies of the IL-2R of lymphocytes, IL$2 \mathrm{R}$ have been identified by the binding of CD25 MAb on selected myelomonocytic cell types. Neither promyelocytes nor more mature neutrophils expressed CD25, but CD25 expression was found on acute myeloid leukemia (AML) ${ }^{1}$ blast cells and increased during culture with PMA, IFN- $\gamma$, the cytokine adult $\mathrm{T}$ cell leukemia-derived factor (ADF), or an impure source of granulocyte-macrophage colony-stimulating factor (GM-CSF) $(6,7)$. Chronic myeloid leukemia (CML) blast cells expressed CD25 only after cell culture, whereas normal bone marrow cells similarly cultured did not express CD25 (8). Basophils purified from CML or normal donors expressed CD25, as did mast cells in tissue sections $(9,10)$. In the instances in which radioligand binding studies were performed, these various myeloid cells had low to intermediate-affinity binding sites for IL-2 (6-10). Monocytes, on the other hand, constitutively express p75; and p55/CD25 is inducible along with highaffinity IL-2R during in vitro activation or diseases associated with monocyte activation (11-14). CD25 expression could be detected on the promyelocytic leukemia cell line HL60 only after treatment with IFN- $\gamma$, which induced monocytic differentiation (11). These studies have delineated distinct patterns of IL-2R subunit expression and offer the possibility that IL-2 activates diverse functions on a variety of cell types.

Eosinophils are a distinctive lineage of granulocytes. Eosinophils normally constitute less than $3 \%$ of circulating leukocytes and are distributed predominantly in submucosal areas of the respiratory and gastrointestinal tracts. In diseases associated with eosinophilia, various $\mathrm{T}$ lymphocyte products are responsible for accelerated production and tissue localization of eosinophils (15). Because IL-2 is a major cytokine product of activated $\mathrm{T}$ lymphocytes, we have evaluated the expression of IL-2R on human eosinophils. Eosinophils express functional IL-2R, and IL-2 can stimulate eosinophils, as evidenced by its capacity to induce receptor-dependent eosinophil migration.

\section{Methods}

Blood donors and eosinophil isolation. Eosinophils were obtained from two donors with the idiopathic hypereosinophilic syndrome (HES) (16), one of whom was being treated with intermittent chlorambucil and hydroxyurea and the other required no therapy. Granulocytes were isolated by dextran sedimentation, centrifugation through Ficoll-Paque (Pharmacia, Piscataway, NJ), and hypotonic lysis of erythrocytes.

1. Abbreviations used in this paper: $\mathrm{ADF}$, adult $\mathrm{T}$ cell leukemia-derived factor; AML, acute myeloid leukemia; CML, chronic myeloid leukemia; ECEF, eosinophil cytotoxicity-enhancing factor; HES, idiopathic hypereosinophilic syndrome; IL-2R, IL-2 receptor(s); LCF, lymphocyte chemoattractant factor; MFI, mean fluorescence intensity; PAF, platelet-activating factor. 
These HES granulocyte preparations contained $74 \pm 13 \%$ eosinophils (mean $\pm \mathrm{SD}, 26$ isolations) with neutrophils as the only contaminating cells. Eosinophils (purity 70\%) were isolated by the Percoll (Pharmacia) gradient technique described below from a patient with filariasis with $12 \%$ blood eosinophilia on no therapy.

From seven normal donors, on no medications and preselected for 2-6\% peripheral blood eosinophilia, eosinophils were isolated on Percoll gradients as described (17). Similar to recently published procedures for depletion of $\mathrm{CD}_{16}{ }^{+}$neutrophils (18), eosinophils were further purified by sequential incubation at $4^{\circ} \mathrm{C}$ with the CD16 MAb 3G8 (purified from supernatant of the hybridoma [19], a gift of Dr. Martin Sanders, Upjohn Co., Kalamazoo, MI) at $2 \mu \mathrm{g}$ per $10^{7}$ granulocytes and goat anti-mouse IgG magnetic beads (Advanced Magnetics, Cambridge, MA) at 20 beads per antibody-coated granulocyte. The final preparations contained $70 \pm 23 \%$ eosinophils (mean $\pm \mathrm{SD}, 6$ isolations) with neutrophils as the only contaminating cells. In selected experiments, immunodepletion of $\mathrm{CD}_{16}{ }^{+}$neutrophils was repeated to yield $100 \%$ pure eosinophils.

Percentage of eosinophils was determined after fast green staining of cytocentrifuge preparations. In selected experiments, monocyte contamination was undetectable (less than one per 500 granulocytes) by $\alpha$-naphthyl esterase staining (Sigma Chemical Co., St. Louis, MO) of purified eosinophils. Binding of the monocyte MAb Leu M3 (CD14; Becton-Dickinson, Mountain View, CA) or T lymphocyte MAb Leu 1 (CD5, Becton-Dickinson) was undetectable in comparison with IgG subclass controls by flow cytometry on purified eosinophils. Viability by trypan blue or propidium iodide exclusion was greater than $98 \%$.

Culture of eosinophils with cytokines. Purified eosinophils were cultured in plastic tissue culture flasks (model 3013; Falcon, Oxnard, CA) at $0.5-1 \times 10^{6} / \mathrm{ml}$ in complete medium: RPMI 1640 (JRH Biosciences, Lenexa, KS) supplemented with $2 \mathrm{mM} \mathrm{L-glutamine,} 100 \mathrm{U} / \mathrm{ml}$ penicillin, $100 \mu \mathrm{g} / \mathrm{ml}$ streptomycin, and 10\% FCS (Gibco, Grand Island, NY). After culture, monocytes and $\mathrm{T}$ lymphocytes were undetectable as assessed above.

Three sources of recombinant human IL-2 were found comparable in effects on eosinophils: (Genzyme, Boston, MA) $2.5 \times 10^{6} \mathrm{U} / \mathrm{mg}$, (R\&D Systems, Minneapolis, MN) $3 \times 10^{6} \mathrm{U} / \mathrm{mg}$, or (Peprotech, Rocky Hill, NJ) $5 \times 10^{6} \mathrm{U} / \mathrm{mg}$. Proliferation of the IL-2-dependent murine cell line CTLL by colorimetric assay (20) confirmed the activities reported by the manufacturers for the IL-2 preparations. Other recombinant human cytokine sources were as follows: affinity-purified ADF (21) (gift of Dr. Junji Yodoi, Virus Research Institute, Kyoto, Japan), GM-CSF (Genzyme) $2.5 \times 10^{5} \mathrm{CFU} / \mu \mathrm{g}$; IFN- $\gamma$ (Genzyme) 2.5 $\times 10^{7} \mathrm{U} / \mathrm{mg}$; IL-1 $\beta$ (Genzyme) $2 \times 10^{8} \mathrm{U} / \mathrm{mg}$; IL-3 (Genzyme) $5 \times 10^{7}$ $\mathrm{U} / \mathrm{mg}$; IL-4 (Genzyme) $1 \times 10^{8} \mathrm{U} / \mathrm{mg}$; IL-5 (Amgen, Thousand Oaks, CA) $1 \times 10^{6} \mathrm{U} / \mathrm{mg}$; IL-6 (Peprotech) ED So $_{\text {with }}$ B9 cells $0.2 \mathrm{ng} / \mathrm{ml}$; and lymphocyte chemoattractant factor (LCF)-containing COS cell supernatant (gift of Dr. David Center, Boston University School of Medicine, Boston, $\mathrm{MA}$ ), $\mathrm{ED}_{50} 1: 10,000$ in eosinophil chemotaxis (the $\mathrm{ED}_{50}$ of purified LCF is $0.1 \mathrm{ng} / \mathrm{ml}$ ) (17).

A partially purified supernatant containing eosinophil cytotoxicityenhancing factor (ECEF) and ADF was generated from the U937 monocytic cell line by stimulation with $100 \mathrm{ng} / \mathrm{ml} \mathrm{PMA} \mathrm{and} 5 \mu \mathrm{g} / \mathrm{ml}$ LPS (butanol extract of Escherichia coli 0127:B8; Sigma Chemical Co.) followed by ammonium sulfate fractionation and phenyl-Sepharose and DEAE ZetaChrom-250 chromatography as detailed previously (22). The material was reconstituted in complete medium at the original volume of the U937 supernatant and used at specified dilutions thereof. Endotoxin activity by Limulus assay (23) was $12 \mathrm{ng} / \mathrm{ml}$ for the purified undiluted material. ECEF activity in enhancing eosinophil killing of Schistosoma mansoni schistosomula was assayed as previously described (22). Two separate preparations were comparable in both ECEF activity and enhancement of eosinophil CD25. Homogenously pure ECEF was isolated from the partially purified supernatant by preparative SDS-PAGE and electroelution of the gel slice of $M_{\mathrm{r}}$ 10,000 as detailed previously (22).

Monoclonal antibodies and flow cytometry analysis. The anti-p55 IL-2R MAb anti-Tac clone 64.1 (CD25) (5) was a gift of Dr. Terry
Strom, Beth Israel Hospital, Boston, MA. The anti-p75 MAb TU27 (24) was a gift of Dr. Kazuo Sugamura, Tohuku University, Sendai, Japan. Anti-HLA class I (clone W6/32 HL) was obtained from SeraLab/Accurate, Westbury, NY. For flow cytometry, FITC-conjugated anti-IL-2R (clone 2A3, CD25) was obtained from Becton Dickinson. Staining was performed as specified by the manufacturer and compared to IgG subclass control FITC MAb staining. For staining of cells binding unconjugated MAb, FITC-conjugated affinity-purified $\mathrm{F}\left(\mathrm{ab}^{\prime}\right)_{2}$ of goat anti-mouse IgG (Tago, Burlingame, CA) was used at a dilution previously determined by titration. Flow cytometry was performed on FACStar ${ }^{\oplus}$ Plus and analyzed by the program Consort 30 (Becton Dickinson). Eosinophils were selectively gated by the combination of forward light scatter, orthogonal light scatter, and autofluorescence. Data were collected on 10,000 cells per sample. Percentage of positive cells was derived from the histogram of MAb of interest by subtraction of the histogram of control MAb. $\triangle$ MFI denotes the difference in mean fluorescence intensity (MFI) between the histogram of interest and that of the control.

Immunoprecipitation of radioiodinated cell surface proteins. Eosinophils harvested from cell culture were washed once in HBSS, resuspended at $10^{8}$ in $1 \mathrm{ml} \mathrm{HBSS}$, transferred to an Iodogen-coated glass tube (Pierce, Rockford, IL), and radioiodinated with $1 \mathrm{mCi}^{125} \mathrm{I}$ (New England Nuclear, Boston, MA). After washing, radioiodinated cells were suspended in $1.5 \mathrm{ml}$ ice-cold HBSS and lysed with an equal volume of $2 \% \mathrm{NP}-40,0.2 \% \mathrm{SDS}, 0.68 \mathrm{M}$ sucrose, $0.15 \mathrm{M} \mathrm{NaCl}, 10 \mathrm{mM}$ phosphate buffer, pH 7.2, $5 \mathrm{mM}$ EDTA, $10 \mathrm{mM} \mathrm{NaF}, 2 \mu \mathrm{M}$ leupeptin, $2 \mu \mathrm{M}$ pepstatin, $0.1 \mathrm{mg} / \mathrm{ml}$ deoxyribonuclease II, $10 \mathrm{mg} / \mathrm{ml} \mathrm{BSA}$, and freshly added $4 \mathrm{mM}$ PMSF (all reagents from Sigma Chemical Co.). After nuclei were pelleted and the supernatant precleared with protein G Sepharose (Zymed, S. San Francisco, CA), the supernatant was sequentially immunoprecipitated with $10 \mu \mathrm{g}$ each of IgG subclass control antibody UPC10 (Organon Teknika-Cappel, West Chester, PA), antiTac, and W6/32, with immunoprecipitates removed by protein $G$ Sepharose after each incubation with antibody. Protein G Sepharosebound immunoprecipitates were solubilized in SDS sample buffer containing 2-mercaptoethanol, electrophoresis was carried out on 10-15\% polyacrylamide gels, and radiolabeled proteins were identified by autoradiography.

Northern blot analysis of $R N A$. For each condition, $10^{8}$ cells were lysed in $2 \mathrm{ml}$ ice-cold $4 \mathrm{M}$ guanidinium isothiocyanate, and RNA was isolated by $\mathrm{CsCl}$ centrifugation as described (25). $15 \mu \mathrm{g}$ RNA from each sample was loaded on a $1 \%$ agarose-formaldehyde gel for electrophoresis. The gel was blotted onto nylon membrane. A cDNA probe for the human IL-2R (p55) consisting of the EcoRI fragment of plasmid pIL2R2 (26) (gift of Dr. Warner Greene, Duke University, Durham, NC) was labeled with $\left.{ }^{32} \mathrm{P}\right] \mathrm{CTP}$ by the random primer method (27), and the blot was hybridized with $1 \times 10^{6} \mathrm{cpm} / \mathrm{ml}$ for $24 \mathrm{~h}$. After hybridization the blot was washed at medium stringency conditions of $0.2 \times \operatorname{SSC}(30$ $\mathrm{mM} \mathrm{NaCl}, 3 \mathrm{mM}$ sodium citrate, $0.05 \%$ sodium pyrophosphate, and $0.1 \%$ sodium lauryl sarkosine) at $56^{\circ} \mathrm{C}$, and hybridization was visualized by autoradiography.

Assessment of IL-2 binding. For saturation binding experiments, ${ }^{125} \mathrm{I}-\mathrm{IL}-2$ (lots with sp act $31-33 \mu \mathrm{Ci} / \mu \mathrm{g}$, nonspecific binding of $4 \%$, and "bindability," or radiolabel capable of specifically binding, of $67-77 \%$ and used within 2 wk of lot date; New England Nuclear) was dialyzed against RPMI 1640, and serial twofold dilutions of radioligand were made in RPMI 1640 supplemented with $10 \mathrm{mg} / \mathrm{ml} \mathrm{BSA} \mathrm{and} 20 \mathrm{mM}$ Hepes buffer, $\mathrm{pH} 7.4$. Each incubation contained $2 \times 10^{6}$ cells in $0.1 \mathrm{ml}$ with $4 \times 10^{-9} \mathrm{M}$ to $4 \times 10^{-12} \mathrm{M}^{125} \mathrm{I}$-IL-2 in siliconized $12 \times 75 \mathrm{~mm}$ glass tubes. For determination of nonspecific binding, a parallel set of tubes also contained dilutions of 150-200-fold excess unlabeled IL-2. After incubations were carried out for $3-4 \mathrm{~h}$ on a rotating table at $4^{\circ} \mathrm{C}$, cell suspensions were transferred to microfuge tubes containing $0.4 \mathrm{ml}$ of a 3:1 mixture of dibutyl phthalate:dionyl phthalate and centrifuged at $12,000 \mathrm{~g}$ for $2 \mathrm{~min}$. The tips of tubes were cut off above the cell pellets and counted in a Searle 1185 gamma counter at $75 \%$ efficiency. Scatchard analysis was performed with the assistance of the LIGAND program (28). Free ligand concentration was considered to be the prod- 


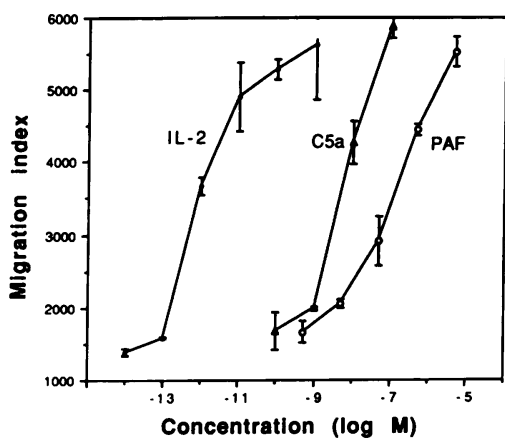

uct of the "bindability" and the difference between radioactivity added and that specifically bound. PHA lymphoblasts derived from PBMC cultured at $10^{6} / \mathrm{ml}$ for $3 \mathrm{~d}$ with $2 \mu \mathrm{g} / \mathrm{ml}$ PHA (Difco, Detroit, MI) were used as a positive control expressing IL-2R.

Assessment of eosinophil migration. Eosinophil migration assays employed our previously described techniques by which eosinophils migrating at several distances into thick nitrocellulose membranes were selectively stained with the fluorescent dye aniline blue and enumerated using fluorescence microscopy and an image analyzer (29). Eosinophils (1-2 $\times 10^{5}$ per well) were suspended in HBSS containing 1 $\mathrm{mg} / \mathrm{ml}$ ovalbumin (Sigma Chemical Co.) and added to the upper wells of microchemotaxis chambers (Neuroprobe, Cabin John, MD), which were separated by a $5-\mu \mathrm{m}$ pore nitrocellulose filter from the chemoattractant in the lower wells. After $60 \mathrm{~min}$ at $37^{\circ} \mathrm{C}$, the filters were stained with aniline blue. Four $25 \times$ fields per well were quantified by counting the fluorescent images of eosinophils at $10-\mu \mathrm{m}$ intervals beginning 10 $\mu \mathrm{m}$ from the upper surface (in general three to four levels within the filter were counted), as described in detail (29), except that the microscope was a Leitz Laborlux S with a $50 \mathrm{~W}$ lamp. Results are expressed as migration index, which is calculated as the sum of the products of cell numbers migrated at each level times the distance for each level counted. Known positive stimuli for eosinophil migration included platelet-activating factor, $\mathrm{L}-\alpha$-phosphatidylcholine $\beta$-acetyl- $\gamma-O$-hexadecyl (PAF; Sigma Chemical Co.), HPLC-purified recombinant human C5a (gift of Dr. Norma Gerard, Beth Israel Hospital, Boston, MA), and CD4-affinity-purified recombinant $\operatorname{LCF}(17,30,31)$.

Statistical analysis. Data are presented as mean \pm SEM. Appropriate two-sample comparisons were performed: Student's $t$ test or paired $t$ test for data that conformed to normal distribution, and Wilcoxon's signed rank test for data that were not normally distributed.

\section{Results}

Migration response of eosinophils to $I L-2$. Because IL-2 induces migration of IL-2R-bearing activated $T$ lymphocytes (32), we evaluated the capacity of IL-2 to stimulate migration of human eosinophils. IL-2 elicited dose-dependent eosinophil migration with $\mathrm{ED}_{50} \sim 10^{-12} \mathrm{M}$ (Fig. 1), equivalent to 0.05 $\mathrm{U} / \mathrm{ml}$ based on IL-2-dependent proliferation assay, and the same $\mathrm{ED}_{\mathrm{so}}$ was found with recombinant IL-2 from three manufacturers. On a molar basis in comparison with the recognized eosinophil chemoattractants $\mathbf{C} 5 \mathrm{a}$ and platelet-activating factor (PAF), IL-2 was 1000-fold more potent (Fig. 1). Eosinophils from all donors tested, six normal and two eosinophilic donors, responded to IL-2 (Table I). In comparisons of eosinophil migration in response to the optimum concentrations for each stimulus, IL-2 at $10^{-10} \mathrm{M}$ was comparably active to LCF (17) at $10^{-10} \mathrm{M}, \mathrm{C} 5 \mathrm{a}$ at $10^{-7} \mathrm{M}$, and PAF at $10^{-6} \mathrm{M}$. In order to determine whether eosinophil migration responses to IL-2 required a chemotactic gradient, we performed checkerboard analyses (33) (Table II). The predominant effect of IL-2 on eosinophil migration was chemokinesis (dose-dependent stimulation of migration independent of a concentration gradient). Thus, IL2 can elicit functional responses in eosinophils, and the responses occur with low concentrations of IL-2 indicative of the involvement of high affinity IL-2R.

We performed antibody blocking experiments to test whether both p75 and p55 Tac subunits of the high affinity IL-2R complex participate in IL-2-induced eosinophil migration. MAb to either p55 (anti-Tac) or p75 (TU27) inhibited the eosinophil chemoattractant response to IL-2 (Fig. 2). IgG subclass controls were not inhibitory. In a total of five experiments, inhibition of IL-2-elicited eosinophil chemotaxis by anti-Tac $(25 \mu \mathrm{g} / \mathrm{ml})$ was $91 \pm 20 \%$, and inhibition by TU27 (25 $\mu \mathrm{g} / \mathrm{ml}$ or $1: 400$ dilution of ascites) was $82 \pm 28 \%$.

Binding of radiolabeled $I L-2$. To characterize the IL-2R present on eosinophils, saturation binding experiments and Scatchard analysis were performed under conditions that would detect high affinity binding (highest radioligand concentration $\left.4 \times 10^{-9} \mathrm{M}\right)$. Minimal specific binding $(<30$ high affinity sites per cell) was detectable with eosinophils from only one of three eosinophilic donors tested. The limited amount of binding did not permit curve fitting to any binding model with statistical confidence. As a positive control expressing high and low affinity IL-2R, ${ }^{125} \mathrm{I}-\mathrm{IL}-2$ binding to PHA-induced lympho-

Table I. Magnitudes of Eosinophil Migration Elicited by IL-2 in Comparison with Other Eosinophil Chemoattractants

\begin{tabular}{|c|c|c|c|c|c|}
\hline \multirow{2}{*}{$\begin{array}{c}\text { Eosinophil } \\
\text { donor }\end{array}$} & \multicolumn{5}{|c|}{ Migration index in response to: } \\
\hline & Medium & $\mathrm{Il}-2\left(10^{-10} \mathrm{M}\right)$ & $\mathrm{C} 5 \mathrm{a}\left(10^{-7} \mathrm{M}\right)$ & $\operatorname{PAF}\left(10^{-6} \mathrm{M}\right)$ & $\operatorname{LCF}\left(10^{-10} \mathrm{M}\right)$ \\
\hline HES 1 & $1196 \pm 70$ & $2044 \pm 167^{\ddagger}$ & $2206 \pm 64^{\S}$ & $2302 \pm 321^{*}$ & $2392 \pm 391^{*}$ \\
\hline HES 2 & $560 \pm 87$ & $1498 \pm 138^{\ddagger}$ & $1700 \pm 56^{5}$ & $725 \pm 123$ & $1260 \pm 72^{\ddagger}$ \\
\hline Normal 1 & $356 \pm 131$ & $3025 \pm 458^{\ddagger}$ & $3917 \pm 271^{\S}$ & $2088 \pm 209^{*}$ & $2449 \pm 793^{*}$ \\
\hline Normal 2 & $1197 \pm 111$ & $1569 \pm 126^{*}$ & ND & $2023 \pm 375$ & $2324 \pm 102^{*}$ \\
\hline Normal 3 & $1012 \pm 200$ & $1592 \pm 256$ & ND & ND & $1712 \pm 94^{*}$ \\
\hline Normal 4 & $118 \pm 42$ & $412 \pm 27^{\ddagger}$ & ND & ND & $586 \pm 175^{*}$ \\
\hline Normal 5 & $1308 \pm 43$ & $5291 \pm 139^{\$}$ & $5886 \pm 170^{\S}$ & $4444 \pm 83^{\S}$ & ND \\
\hline Normal 6 & $369 \pm 45$ & $551 \pm 49^{*}$ & $652 \pm 70^{*}$ & $320 \pm 34$ & ND \\
\hline
\end{tabular}

Migration responses (migration index \pm SEM of triplicates) of eosinophils from six normal and two HES donors to control medium and to maximally active concentrations of IL-2 and other eosinophil chemoattractants. ${ }^{*} P<0.05,{ }^{\ddagger} P<0.005,8<0.0005$ in comparison to medium. 
Table II. Checkerboard Analysis of Eosinophil Migration Stimulated by IL-2

\begin{tabular}{|c|c|c|c|c|c|}
\hline \multirow{3}{*}{$\begin{array}{c}\mathrm{IL}-2\left(10^{-12} \mathrm{M}\right) \\
\text { (in lower chamber) }\end{array}$} & \multicolumn{5}{|c|}{ Migration index (mean \pm SEM of triplicates) } \\
\hline & \multicolumn{5}{|c|}{ IL-2 $\left(10^{-12} \mathrm{M}\right)$ in upper chamber } \\
\hline & 0 & 0.4 & 2 & 10 & 50 \\
\hline 0 & $2876 \pm 377$ & $2882 \pm 189$ & ND & ND & ND \\
\hline 0.4 & $3545 \pm 163$ & $3459 \pm 166$ & $3096 \pm 438$ & ND & ND \\
\hline 2 & $3667 \pm 187$ & $3428 \pm 298$ & $3836 \pm 34$ & ND & ND \\
\hline 10 & $4372 \pm 382$ & ND & ND & $4214 \pm 271$ & ND \\
\hline 50 & $4998 \pm 425$ & ND & ND & ND & $4730 \pm 344$ \\
\hline
\end{tabular}

Results are representative of two similar experiments with eosinophils from two normal donors.

blasts (mean $66 \% \mathrm{CD} 25^{+}$by flow cytometry) revealed a curvilinear Scatchard plot which could be fitted by two affinities of binding sites: $800 \pm 300$ high affinity sites per cell with an apparent $K_{\mathrm{d}}$ of $3 \pm 2 \times 10^{-11} \mathrm{M}$, and $11,000 \pm 2,000$ low affinity sites per cell with an apparent $K_{\mathrm{d}}$ of $6 \pm 1 \times 10^{-9} \mathrm{M}(n=3)$. Thus, minimal numbers of high affinity IL-2R could be detected on eosinophils of one of three donors.

Eosinophil IL-2R expression analyzed by flow cytometry. Expression of p55 was measured by flow cytometry with CD25 MAb. Eosinophils from all donors expressed low levels of CD25: mean (range) in percentage $\mathrm{CD} 25^{+}$were $28 \%(9-54 \%)$ for HES eosinophils ( $n=11$ from 2 donors), $38 \%$ for eosinophils from a filariasis patient, and 9\% (4-17\%) for eosinophils from normal donors $(n=3$ ); mean (range) in $\triangle$ MFI were 8.9 (4.7-15.5) for HES eosinophils, 4.3 for eosinophils from a filariasis patient, and $2.4(1.8-2.8)$ for normal eosinophils. In contrast, expression of p75 measured by flow cytometry with TU27 MAb was not detectable on eosinophils from normal or eosinophilic donors $(n=8)$.

Identity of the surface antigen which binds CD25 MAb as p55 Tac. To ascertain that the eosinophil surface antigen recognized by CD25 MAb was the p55 Tac subunit of IL-2R, we performed radioimmunoprecipitation. HES eosinophils, purified to $100 \%$ with two cycles of immunodepletion of CD16 $6^{+}$ neutrophils, were cultured with a partially purified U937 supernatant that enhances CD25 expression (see below). By flow cytometry, eosinophils demonstrated a unimodal pattern of 2A3 CD25 MAb staining (Fig. $3 A$ ), and eosinophils were $56 \%$ $\mathrm{CD}^{2} 5^{+}$. On analysis of cell surface radioiodinated eosinophils (Fig. 3 B), immunoprecipitation with subclass IgG control

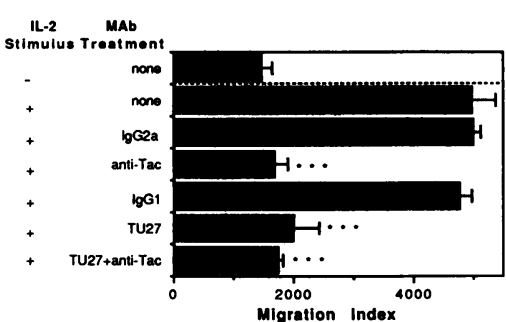

Figure 2. Inhibition of the eosinophil chemoattractant response to IL2 by MAbs against $\mathrm{p} 55$ and p75 IL-2R. Migration responses (migration index \pm SEM of triplicates) of eosinophils from a normal donor to medium or $10^{-10}$ M IL-2 are indicated by

$(-)$ or (+), respectively. The indicated MAbs at $25 \mu \mathrm{g} / \mathrm{ml}$ were added to the eosinophils immediately prior to their addition to the upper wells of the microchemotaxis chambers. Control $\mathrm{IgG}_{2 \mathrm{a}}$ and $\mathrm{IgG}_{1}$ were UPC10 and MOPC21, respectively. ${ }^{* * *} P<0.0005$ in comparison to IL-2-stimulated migration in the absence of antibody, Student's $t$ test. (lane 1 ) revealed only radioactive material migrating with the dye front, and immunoprecipitation with anti-Tac yielded a major band of $M_{\mathrm{r}} 55,000$ (lane 2), consistent with the p55 subunit of IL-2R.

mRNA for p55 Tac in eosinophils. To investigate mechanisms of IL-2R expression by eosinophils, we examined mRNA by Northern blot analysis. mRNA for p55 Tac was present in freshly isolated HES eosinophils (Fig. 4, lane 1). PBMC isolated from the same blood specimen contained much less mRNA for p55 IL-2R (lane 2), similar to findings with normal PBMC in which p55 mRNA was minimal without in vitro stimulation (1). The lesser $m R N A$ expression in PBMC also established that the finding of p55 mRNA in purified eosinophils was not attributable to mononuclear cell contamination (500-cell differential counts of the purified eosinophils contained no mononuclear cells). The two sizes of p55 mRNA in eosinophils were at the same positions as the 3.5 and 1.4-kb species of p55 mRNA in PHA-induced lymphoblasts in a sepa-
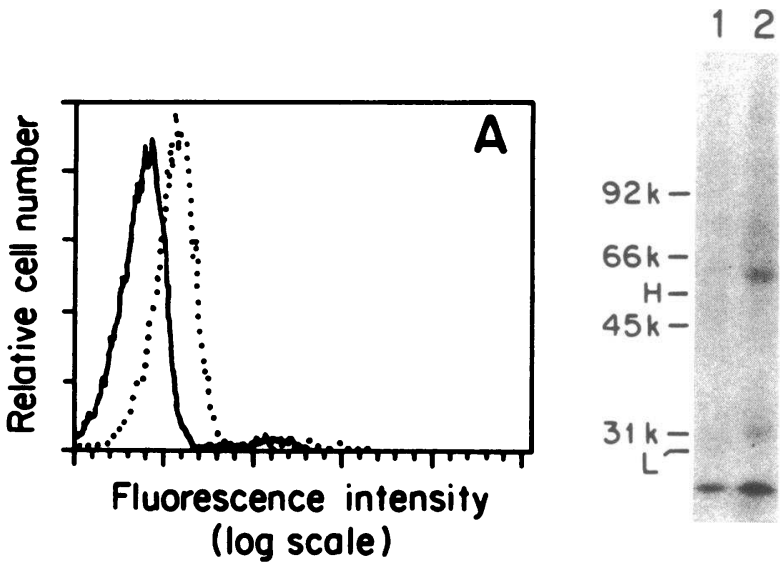

Figure 3. Immunoprecipitation of p55 (Tac/CD25). Eosinophils isolated at $100 \%$ purity from HES donor 2 were cultured for $48 \mathrm{~h}$ with partially purified U937 supernatant at 1:7 dilution. $(A)$ Histograms of flow cytometry analysis after staining with FITC-anti-IL-2R 2A3 (dotted line) or subclass control FITC-MAb (solid line). Subtraction of the two histograms calculated $56 \% \mathrm{CD}^{2} 5^{+}$. (B) Immunoprecipitation and gel electrophoresis of radioiodinated cell surface proteins was performed as described in Methods with $\mathrm{IgG}_{2 \mathrm{a}}$ myeloma protein UPC10 (lane 1 ) and CD25 MAb anti-Tac (lane 2). The positions of immunoglobulin heavy chains $(H)\left(M_{\mathrm{r}} \sim 50,000\right)$, immunoglobulin light chains $(L)\left(M_{\mathrm{r}} \sim 25,000\right)$, and molecular size standards (BioRad, Richmond, CA) are indicated. 


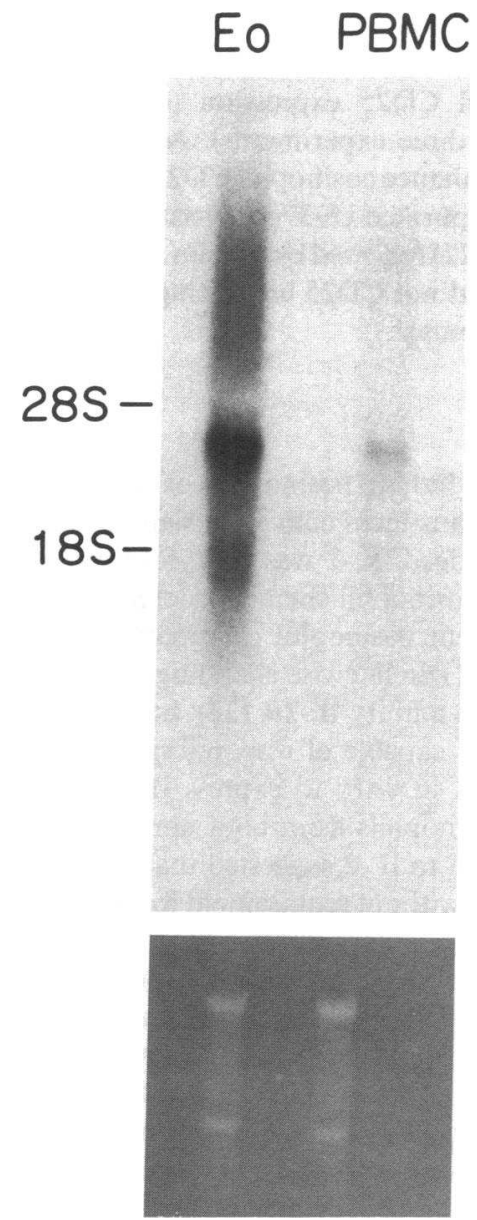

Figure 4. Expression of mRNA for p55 IL-2R by eosinophils and PBMC. RNA extracted from eosinophils (63\% purity with neutrophils as the only contaminating cells) and PBMC (50\% lymphocytes, $34 \%$ monocytes, $16 \%$ neutrophils) isolated from the same blood specimen from HES donor 1 were analyzed by Northern blot probed with p55 IL-2R cDNA. The positions of $28 \mathrm{~S}$ and $18 \mathrm{~S}$ RNA visualized by ethidium staining (shown in lower panel to confirm uniform loading of RNA) are indicated.

rate experiment (the two RNA species result from alternative polyadenylation signals [26]). Eosinophils from the second HES donor similarly contained mRNA for p55 IL-2R (not shown).

Lack of effect of pharmacologic agents on p55 IL-2R expression. We examined agents that might upregulate p55 IL-2R on eosinophils. Pharmacologic agents that activate p55 IL-2R expression on lymphocytes include PMA, which directly activates protein kinase $C$, the calcium ionophore $A 23187$, and forskolin, which elevates cAMP by activation of adenylate cyclase $(1,34,35)$. None of these agents enhanced eosinophil p55 IL-2R, measured by flow cytometry after staining for CD25 (data not shown). Because PMA is a degranulating stimulus that impairs the viability of eosinophils at high concentrations, a range of concentrations $\left(10^{-10}\right.$ to $\left.10^{-8} \mathrm{M}\right)$ and time points (1 to $24 \mathrm{~h}$ ) were examined with negative results. Similarly, A23187 $\left(10^{-9}\right.$ to $\left.10^{-7} \mathrm{M}\right)$ and forskolin $\left(10^{-5} \mathrm{M}\right)$ were without effect. In lymphocytes, phorbol diester and calcium ionophore are synergistic in activation of p55 IL-2R expression (1), but this was not found when eosinophils were cultured with combinations of $\mathrm{A} 23187\left(10^{-9}\right.$ to $\left.10^{-8} \mathrm{M}\right)$ and PMA $\left(10^{-10}\right.$ to $\left.10^{-9} \mathrm{M}\right)$ (data not shown). In contrast to its effect on monocytes (12), LPS $(5 \mu \mathrm{g} / \mathrm{ml})$ did not enhance eosinophil p55 IL-2R expression (data not shown).

Variation in p55 IL-2R expression on eosinophils during culture with cytokines. To understand whether IL-2R expression might be immunologically regulated, we examined eosinophils from normal and eosinophilic donors before and after culture with medium or cytokines known to be active on eosinophils (Fig. 5). Before cell culture, eosinophils varied in CD25 expression as noted above. The cytokines evaluated were the colony-stimulating factors active on eosinophils (GM-CSF, IL3 , and IL-5), IFN- $\gamma$, LCF, and a partially purified U937 supernatant which contained ECEF and ADF (22). The only material that consistently enhanced CD25 on HES eosinophils during 24-48-h culture was the U937-derived material. Comparison of CD25 expression on eosinophils cultured in medium versus U937-derived factor was significant whether analyzed as percentage $\mathrm{CD}_{2} 5^{+}(P<0.001$, the increase in percentage CD25 $5^{+}$was $\left.22 \pm 3, n=13\right)$ or as MFI $(P<0.005$, the increase in MFI was 5.1 \pm 1.0 ). The capability for enhanced p55 IL-2R expression by normal eosinophils was evident in experiments with the U937-derived activity: after culture eosinophils from three normal donors were 63,38 , and $14 \% \mathrm{CD} 25^{+}$. GMCSF and LCF each increased eosinophil CD25 expression in some but not all experiments, and overall the increases were statistically significant. IFN- $\gamma$ significantly depressed CD25 expression in comparison to the effects of culture in medium alone, but the magnitude of the effect was small. IL-3 increased CD25 in individual experiments, sometimes dramatically, but overall the effects were not statistically significant. No trends were evident in the effects of IL-5. Other cytokines that did not influence eosinophil CD25 expression included IL-1 $\beta\left(10^{-12}\right.$ to $\left.10^{-10} \mathrm{M}\right)$, IL-2 $\left(10^{-10}\right.$ to $\left.10^{-8} \mathrm{M}\right)$, IL-4 $\left(10^{-11}\right.$ to $\left.10^{-8} \mathrm{M}\right)$, IL-6 $\left(10^{-11}\right.$ to $\left.10^{-9} \mathrm{M}\right)$, combination of IL-5 with IL-2, and combination of IL-5 or GM-CSF with LCF (data not shown, each tested in a minimum of two experiments). When HES eosinophils

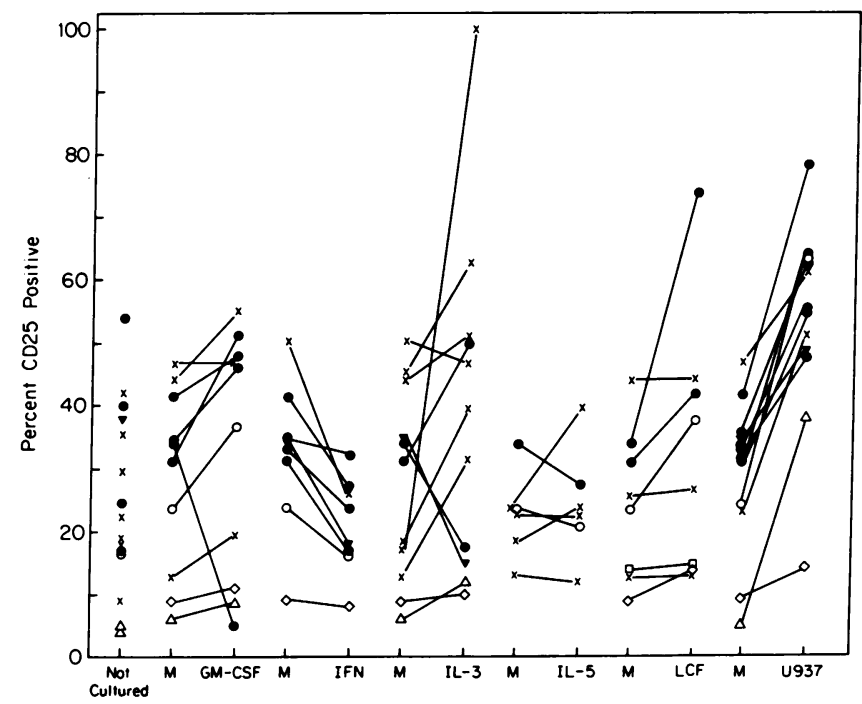

Figure 5. Expression of p55/CD25 during culture of eosinophils with cytokines. Eosinophils isolated from normal $(0, \Delta, \square, \diamond)$, HES $(\times, \bullet)$, or filariasis $(\nabla)$ donors were analyzed by flow cytometry for percentage $\mathrm{CD}^{2} 5^{+}$(results in column labeled "not cultured"). The cell preparations were then cultured for $24-48 \mathrm{~h}$ in complete medium $(M)$ or the indicated cytokines, and the percentage $\mathrm{CD} 25^{+}$determined. Cytokine concentrations were GM-CSF, $1 \times 10^{-11} \mathrm{M} ; \mathrm{IFN}-\gamma, 1 \times 10^{-10} \mathrm{M} ; \mathrm{IL}-3$, $1-2 \times 10^{-11} \mathrm{M} ; \mathrm{IL}-5,1-5 \times 10^{-11} \mathrm{M} ; \mathrm{LCF}, 1: 30$ dilution of COS supernatant; and $U 937$, partially purified supernatant at 1:2 dilution. Statistical differences from medium treated were U937 treated at $P$ $<0.001$, IFN treated or GM-CSF treated at $P<0.01$, LCF treated at $P<0.05$; data from eosinophilic and normal donors were analyzed together since changes were in the same direction for each group. 
were maintained in culture with murine $3 \mathrm{~T} 3$ fibroblasts and either GM-CSF, IL-3, or IL-5 by methods previously reported (36), eosinophil CD25 expression declined to less than $20 \%$ $\mathrm{CD}^{2} 5^{+}$at 3-6 d of culture (data not shown, six experiments).

Characterization of p55 IL-2R enhancement by U937-derived factor. The time courses of changes in CD25 during culture with U937-derived factor, GM-CSF, IFN- $\gamma$, and medium were compared (Fig. 6). The enhancement by the U937-derived factor was detectable at $24 \mathrm{~h}$ and increased further by 48 h. Flow cytometry histograms are shown in $A$ to illustrate the change in eosinophil CD25 during culture with U937-derived factor. IFN- $\gamma$ persistently depressed CD25. The magnitude of CD25 expression during culture with GM-CSF or medium alone was similar to that on freshly isolated eosinophils. These experiments were not extended beyond $48 \mathrm{~h}$ because of the decline in eosinophil viability when cultured in medium without cytokines.

The partially purified U937 supernatant was selected because it contained ECEF. ADF, a 13,000- $M_{\mathrm{r}}$ factor that shares $\mathrm{NH}_{2}$-terminal sequence homology with $10,000-M_{\mathrm{r}} \mathrm{ECEF}$, is also present in this preparation (Silberstein, D. S., unpublished results). ECEF activity defined by eosinophil-mediated killing of schistosomula paralleled CD25 enhancing activity when dilutions of the partially purified U937 supernatant were tested in the two assays (data not shown). To determine whether ECEF or ADF was responsible for CD25 enhancement by the partially purified supernatant, we tested pure ECEF eluted from SDS-polyacrylamide gels and recombinant purified
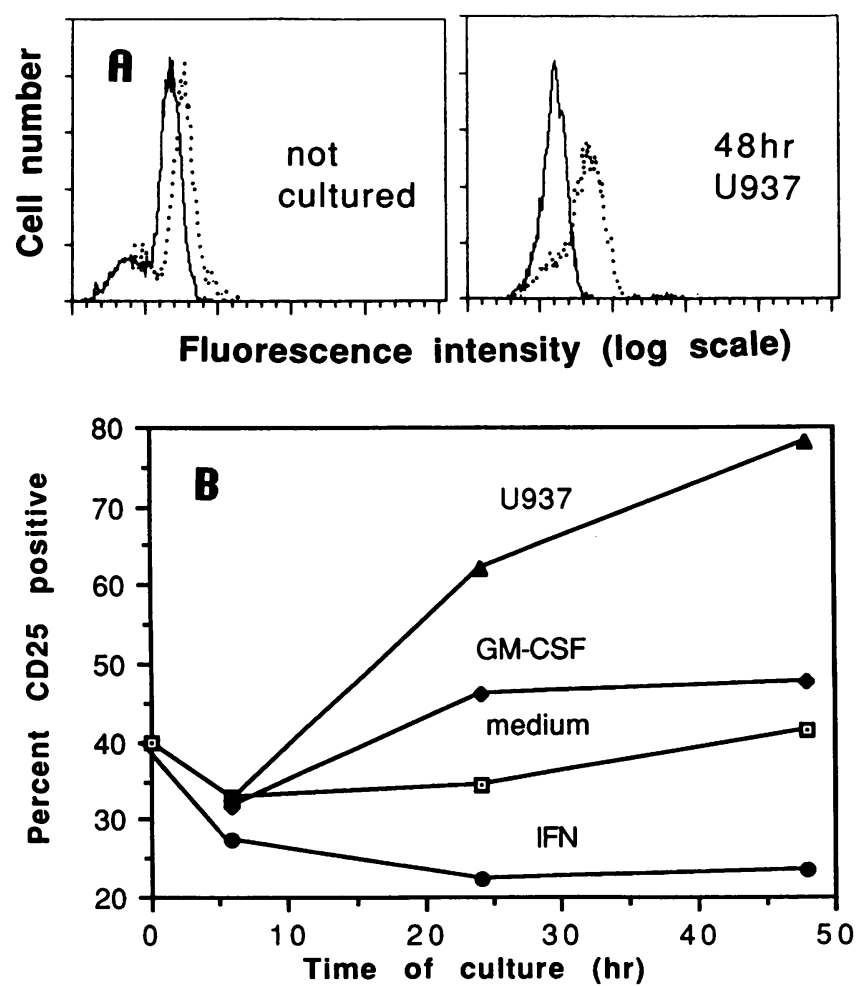

Figure 6. Time course of changes in p55/CD25 expression during culture of eosinophils. Eosinophils (76\% purity) from HES donor 2 were cultured for the indicated times with complete medium (ם); GM-CSF, $1 \times 10^{-11} \mathrm{M}(\bullet)$; IFN- $\gamma, 1 \times 10^{-10} \mathrm{M}(\bullet)$; or U937, partially purified supernatant at $1: 2$ dilution $(\Lambda)$, and percentage $\mathrm{CD}^{2} 5^{+}(B)$ was determined by flow cytometry (illustrated in $A$ ).
ADF. Neither ECEF (up to $20 \times$ the $\mathrm{ED}_{50}$ for eosinophil-mediated schistosomula killing) nor $\operatorname{ADF}\left(10^{-5}\right.$ to $\left.10^{-10} \mathrm{M}\right)$ individually influenced eosinophil CD25 expression (data not shown, each tested in two to three experiments). As further evidence that ECEF does not enhance eosinophil CD25 expression, treatment of the partially purified U937 supernatant with rabbit antibody against $\operatorname{ECEF}(22)$ followed by protein A Sepharose depleted ECEF activity but not CD25 enhancing activity (data not shown, two experiments).

\section{Discussion}

Our findings demonstrate that human eosinophils express IL$2 \mathrm{R}$ and that binding of IL- 2 transduces activating signals that elicit eosinophil migration. Indeed, IL-2 was 1000 -fold more potent on a molar basis as an eosinophil chemoattractant than two currently recognized potent eosinophil chemoattractant agents, PAF and C5a (30). IL-2 can likewise elicit migration of T lymphocytes expressing high affinity IL-2R (32); but unlike eosinophils, lymphocytes were capable of responding to IL-2 only after they were activated in vitro to express IL-2R. In contrast, the capability of eosinophils from both normal and eosinophilic donors to respond to IL-2 suggested that eosinophils express functional IL-2R without requirement for in vitro activation.

Flow cytometric analysis of eosinophil expression of the two IL-2R subunit proteins failed to detect expression of p75 but did detect low levels of $\mathrm{p} 55 / \mathrm{CD} 25$, and the magnitude of CD25 expression was greater on eosinophils derived from eosinophilic donors than for normal donors. Expression of p55 was also demonstrable by radioimmunoprecipitation, and eosinophils contained mRNA for p55. While quantitation of CD25 expression by flow cytometry was based on fluorescence intensity in excess of control after MAb staining, two findings suggest that most, if not all eosinophils, express functional IL$2 \mathrm{R}$. First, CD25 expression with the most highly purified eosinophils (Fig. $3 \mathrm{~A}$ ) was not a bimodal pattern indicative of positive and negative eosinophils; rather, CD25 staining yielded a unimodal histogram that overlapped the control histogram. Second, chemoattractant responses indicated that migration was elicited on eosinophils expressing minimal IL-2R. Our chemotaxis method evaluated not simply the most rapidly migrating cells at the front of elicited migration but rather quantitated the migration of the entire population of eosinophils that had penetrated the filter. Significant eosinophil migration in response to IL-2 was found even with normal eosinophils that had little CD25 expression detected by flow cytometry. Moreover, the numbers of migrating eosinophils (as measured by migration index) with IL-2 were comparable to those found with PAF and C5a, for which large numbers of receptors are present on eosinophils (31). The magnitudes of eosinophil chemoattractant responses to IL-2 indicate that expression of IL$2 \mathrm{R}$ was present on a preponderance of eosinophils.

Although eosinophils had low to undetectable numbers of high affinity binding sites for ${ }^{125} \mathrm{I}-\mathrm{IL}-2$, several findings suggest that high affinity IL-2R mediate the response of eosinophils to IL-2. First, the $\mathrm{ED}_{50}$ for IL-2 as an eosinophil chemoattractant was $10^{-12} \mathrm{M}(0.05 \mathrm{U} / \mathrm{ml})$, typical for cells bearing high affinity IL-2R $(1,12,32)$. Second, the capacity of MAb against either p55 or p75 IL-2R to inhibit the chemoattractant response of eosinophils to IL-2 suggests that the functional IL-2R is composed of p55 and p75 in heterodimeric complexes, which on 
lymphocytes constitute the high affinity IL-2R $(2,4,37)$. There are analogous situations (specifically, with $\mathrm{CD}^{+} \mathrm{T}$ lymphocytes and $\mathrm{CD}_{16}{ }^{+} \mathrm{NK}$ cells) in which investigators have implicated responses mediated by high affinity IL-2R in which one of the subunits was not demonstrable: MAb to p55 inhibited IL-2-dependent cytotoxicity, although p55 was undetectable on these cells by flow cytometry, and high affinity IL-2 binding sites were very low (37-39).

Because p55/CD25 expression was greater on eosinophils from eosinophilic diseases compared with normal eosinophils, we evaluated stimuli that might modulate eosinophil CD25 expression including cytokines known to have activities for eosinophils. GM-CSF and IL-3, colony-stimulating factors with similar activities for eosinophils, did not consistently upregulate eosinophil CD25, although the increases in CD25 with GM-CSF attained statistical significance. The results with IL-3 are of interest in relationship to the report that IL-3 enhanced p55 IL-2R on murine hemopoietic cell lines (40). LCF, a chemoattractant for $\mathrm{CD}^{+}$leukocytes including eosinophils (17), upregulated CD25 expression of $\mathrm{CD}^{+}$lymphocytes (41) and significantly but minimally increased eosinophil CD25 under the conditions examined. A number of cytokines that failed to enhance CD25 on eosinophils do so on other cell types: IFN- $\gamma$ enhanced monocyte p55/CD25; IL-2 and IL-5 enhanced lymphocyte $\mathrm{p} 55 / \mathrm{CD} 25(1,11,12,42,43)$. The dichotomy between monocytes and eosinophils with regard to the effect of IFN- $\gamma$ is of interest, as with both eosinophils and monocytes IFN- $\gamma$ stimulates cytotoxicity (44) and induces HLA-DR (Rand, T. H., and P. F. Weller, unpublished results). The effects of pharmacologic agents suggest that intracellular events responsible for enhancement of p55 IL-2R may be quite different in eosinophils from those in lymphocytes: PMA, which activates protein kinase C, A23187, which increases cytoplasmic calcium, and forskolin, which increases cAMP are activators of transcription of mRNA for p55 in lymphocytes $(1,34,35)$ but did not influence p55/CD25 expression of eosinophils.

The only stimulus that consistently enhanced p55/CD25 expression was an activity partially purified from stimulated U937 cells. Endotoxin was not responsible for the U937 supernatant activity: LPS did not enhance eosinophil CD25 expression either by itself or after addition to the partially purified U937 supernatant. The U937-derived material was purified based on its ECEF activity, defined by eosinophil killing of target parasites. When the chromatographically purified material is subjected to its final purification step (elution from SDSpolyacrylamide gels) there is considerable loss of ECEF activity (22); it is conceivable that loss of CD25-enhancing activity may have occurred as well. Against this interpretation, however, was the finding that an antibody that neutralizes ECEF did not decrease the CD25-enhancing activity of the U937-derived material. We also considered the possibility that a molecularly related factor ADF was present in the partially purified U937 supernatant. ADF enhanced $\mathrm{p} 55 / \mathrm{CD} 25$ expression on a variety of lymphoid cells and AML cells $(7,21)$. The $13,000-M_{\mathrm{r}} \mathrm{ADF}$ is similar physicochemically to the $10,000-M_{\mathrm{r}}$ ECEF, and they share $\mathrm{NH}_{2}$-terminal amino acid sequence homology with thioredoxin $(21,22)$. However, neither pure ECEF nor ADF upregulated eosinophil CD25; hence, the biologic activity from U937 cells remains unidentified.

There are a number of immunopathologic correlations between IL-2 and eosinophils, but the immunologic roles served by IL-2R on eosinophils are not certain. Elevated serum levels of soluble IL-2R have been noted in patients with HES, although these soluble IL-2R may have been lymphocyte derived (45). In view of the activity of IL-2 as a chemoattractant, IL-2 could contribute to the recruitment of eosinophils to sites of lymphocyte activation. Concomitant infiltration of eosinophils and activated IL-2R-bearing lymphocytes have been noted, for instance, in the respiratory tract in chronic asthma (46). IL-2 administered in the bladder, peritoneum, and pleural space has been followed by local eosinophilia, but repeated high doses of IL-2 were used over days to weeks, which also resulted in blood eosinophilia (47-49). Systemic administration of IL-2 frequently results in eosinophilia, and the blood eosinophils have an activated phenotype (50), findings that could be secondary to IL- 5 produced by IL-2-activated lymphocytes (51). While the extent to which IL-2 mediates local eosinophil accumulation in the above circumstances is unclear, IL-2 may stimulate functional responses of eosinophils in addition to migration, akin to GM-CSF and IL-5, which affect mature leukocyte function and have chemoattractant activities. Previous reports indicated that IL-2 affected the migration of murine eosinophils but did not enhance degranulation or cytotoxicity of human or rodent eosinophils $(44,52-54)$. The recognition that human eosinophils express functional IL-2R demonstrates further similarities among monocytes, lymphocytes, and eosinophils in that each can express CD4 and HLADR as well as IL-2R $(17,-36)$. The presence of high affinity IL-2R on eosinophils provides a mechanism by which eosinophils can be stimulated coordinately with IL-2R-bearing mononuclear cells in pathologic reactions in which infiltrating eosinophils are found along with activated lymphocytes.

\section{Acknowledgments}

The authors gratefully acknowledge the research flow cytometry skills of Ms. Claudia Cabral, the statistical advice of Dr. Bernard Ransil, the laboratory assistance of Ms. Tonya Barrett, the assistance of Dr. Gur Notani in CTLL assay, the initial observations of Dr. Daniel Lucey, the continued enthusiasm and support of Dr. Anne Nicholson-Weller, the gift of MAb by Dr. Kazuo Sugamura, and of cytokine by Dr. Junji Yodoi.

Research was supported by AI-20241, AI-28525, and HL-44846 from the National Institutes of Health, and the MWV Leukocyte Research Fund. Dr. Rand was supported by an NIH Training Grant AI07061 and subsequently is the recipient of a Fellowship in Immunology from The Irvington Institute for Medical Research. Dr. Kornfeld is the recipient of an American Lung Association Career Investigator Award.

\section{References}

1. Waldmann, T. A. 1989. The multi-subunit interleukin-2 receptor. Annu. Rev. Biochem. 58:875-911.

2. Robb, R. J., W. C. Greene, and C. M. Rusk. 1984. Low and high affinity cellular receptors for interleukin 2. Implications for the level of Tac antigen. J. Exp. Med. 160:1126-1146.

3. Tsudo, M., R. W. Kozak, C. K. Goldman, and T. A. Waldmann 1986 Demonstration of a non-Tac peptide that binds interleukin 2: A potential participant in a multichain interleukin 2 receptor complex. Proc. Natl. Acad. Sci. USA. 83:9694-9698.

4. Wang H.-M., and K. A. Smith. 1987. The interleukin 2 receptor. Functional consequences of its bimolecular structure. J. Exp. Med. 166:1055-1069.

5. Uchiyama, T., S. Broder, and T. A. Waldmann. 1981. A monoclonal antibody (anti-Tac) reactive with activated and functionally mature human $T$ cells. I. Production of anti-Tac monoclonal antibody and distribution of Tac(+) cells. $J$. Immunol. 126:1393-1403.

6. Armitage, R. J., A. P. Lai, P. J. Roberts, and J. C. Cawley. 1986. Certain myeloid cells possess receptors for interleukin-2. Br. J. Haematol. 64:799-807. 
7. Yamamoto, S., T. Hattori, M. Matsuoka, T. Ishii, N. Asou, M. Okada, Y. Tagaya, J. Yodoi, and K. Takatsuki. 1986. Induction of Tac antigen and proliferation of myeloid leukemic cells by ATL-derived factor: comparison with other agents that promote differentiation of human myeloid or monocytic leukemic cells. Blood. 67:1714-1720.

8. Visani, G., R. Delwel, I. Touw, F. Bot, and B. Lowenberg. 1987. Membrane receptors for interleukin 2 on hematopoietic precursors in chronic myeloid leukemia. Blood. 69:1 182-1187.

9. Stockinger, H., P. Valent, O. Majdic, P. Bettelheim, and W. Knapp. 1990. Human blood basophils synthesize interleukin-2 binding sites. Blood. 75:1820 1826.

10. Maggiano, N., F. Colotta, F. Castellino, R. Ricci, S. Valitutti, L. M. Larocca, and $P$. Musiani. 1990. Interleukin-2 receptor expression in human mast cells and basophils. Int. Arch. Allergy Appl. Immunol. 91:8-14.

11. Herrmann, F., S. A. Cannistra, H. Levine, and J. D. Griffin. 1985. Expression of interleukin 2 receptors and binding of interleukin 2 by gamma interferoninduced human leukemic and normal monocytic cells. J. Exp. Med. 162:11111116.

12. Holter, W., C. K. Goldman, L. Casabo, D. L. Nelson, W. C. Greene, and T. A. Waldmann. 1987: Expression of functional IL 2 receptors by lipopolysaccharide and interferon stimulated human monocytes. J. Immunol. 138:29172922.

13. Toosi, Z., J. R. Sedor, J. P. Lapurga, R. J. Ondash, and J. J. Ellner. 1990. Expression of functional interleukin 2 receptors by peripheral blood monocytes from patients with active pulmonary tuberculosis. J. Clin. Invest. 85:1777-1784.

14. Espinoza-Delgado, I., J. R. Ortaldo, R. Winkler-Pickett, K. Sugamura, L. Varesio, and D. L. Longo. 1990. Expression and role of p 75 interleukin 2 receptor on human monocytes. J. Exp. Med. 171:1821-1826.

15. Colley, D. G. 1980. Lymphokine-related eosinophil responses. In Lymphokine Reports. E. Pick, editor. Academic Press, New York. 1:133-155.

16. Fauci, A. S., J. B. Harley, W. C. Roberts, V. J. Ferrans, H. R. Gralnick, and B. H. Bjornson. 1982. The idiopathic hypereosinophilic syndrome. Clinical pathophysiologic, and therapeutic considerations. Ann. Int. Med. 97:78-92.

17. Rand, T. H., W. W. Cruikshank, D. M. Center, and P. F. Weller. 1991 CD4-mediated stimulation of human eosinophils. Lymphocyte chemoattractant factor and other CD4-binding ligands elicit eosinophil migration. J. Exp. Med. 173:1521-1528

18. Hansel, T. T., J. D. Pound, D. Pilling G. D. Kitas, M Salmon, T. A Gentle, S. S. Lee, and R. A. Thompson. 1989. Purification of human blood eosinophils by negative selection using immunomagnetic beads. J. Immunol. Methods 122:97-103.

19. Fleit, H. B., S. D. Wright, and J. C. Unkeless. 1982. Human neutrophil Fc receptor distribution and structure. Proc. Natl. Acad. Sci. USA. 79:3275-3279.

20. Mossman, T. R., and T. A. T. Fong. 1989. Specific assays for cytokine production by T cells. J. Immunol. Methods. 116:151-158.

21. Tagaya, Y., Y. Maeda, H. Matsui, J. Hamuro, N. Brown, K. Arai, T. Yokota, H. Wakasugi, and J. Yodoi. 1989. ATL-derived factor (ADF), an IL-2 receptor/Tac inducer homologous to thioredoxin; possible involvement of dithiol-reduction in the IL-2 receptor induction. EMBO (Eur. Mol. Biol. Organ.) J. 8:757-764.

22. Silberstein, D. S., M. H. Ali, S. L. Baker, and J. R. David. 1989. Human eosinophil cytotoxicity-enhancing factor. Purification, physical characteristics, and partial amino acid sequence of an active polypeptide. J. Immunol. 143:979983.

23. Milton, D. K., R. J. Gere, H. A. Feldman, and I. A. Greaves, 1990. Endotoxin measurement: Aerosol sampling and application of a new Limulus method. Am. Ind. Hyg. Assoc. J. 51:331-337.

24. Takeshita, T., Y. Goto, K. Tada, K. Nagata, H. Asao, and K. Sugamura 1989. Monoclonal antibody defining a molecule possibly identical to the p75 subunit of interleukin 2 receptor. J. Exp. Med. 169:1323-1332.

25. Ausubel, F. M., F. M. Brent, R. E. Kingston, D. D. Moore, J. G. Seidman J. A. Smith, and K. Struhl. 1989. Current Protocols in Molecular Biology. John Wiley \& Sons, New York. 4.2.1-4.2.4.

26. Leonard, W. J., J. M. Depper, G. R. Crabtree, S. Rudikoff, J. Pumphrey, R. J. Robb, M. Kronke, P. B. Svetlik, N. J. Peffer, T. A. Waldmann, and W. C. Greene. 1984. Molecular cloning and expression of cDNAs for the human interleukin 2 receptor. Nature (Lond.). 311:626-631.

27. Feinberg, A. P., and B. Vogelstein. 1983. A technique for radiolabeling DNA restriction endonuclease fragments to high specific activity. Anal. Biochem. 132:6-13.

28. Munson, P. J., and D. Rodbard. 1980. LIGAND a versatile computerized approach for characterization of ligand binding systems. Anal. Biochem. 107:220-239.

29. McCrone, E. L., D. R. Lucey, and P. F. Weller. 1988. Fluorescent staining for leukocyte chemotaxis: eosinophil-specific staining with aniline blue. J. Immunol. Methods 114:79-88.

30. Wardlaw, A. J., R. Moqbel, O. Cromwell, and A. B. Kay. 1986. Plateletactivating factor. Potent chemotactic and chemokinetic factor for human eosinophils. J. Clin. Invest. 78:1701-1706.
31. Gerard, N. P., M. K. Hodges, J. M. Drazen, P. F. Weller, and C. Gerard. 1989. Characterization of a receptor for C5a anaphylatoxin on human eosinophils. J. Biol. Chem. 264:1760-1766.

32. Kornfeld, H., J. S. Berman, D. J. Beer, and D. M. Center. 1985. Induction of human T lymphocyte motility by interleukin 2. J. Immunol. 134:3887-3890.

33. Zigmond, S. H., and J. G. Hirsch. 1973. Leukocyte locomotion and chemotaxis. New methods for evaluation, and demonstration of a cell-derived chemotactic factor. J. Exp. Med. 137:387-410.

34. Depper, J. M., W. J. Leonard, M. Kronke, P. D. Noguchi, R. E. Cunningham, T. A. Waldmann, and W. C. Greene. 1984. Regulation of interleukin 2 receptor expression: effects of phorbol diester, phospholipase $\mathrm{C}$, and reexposure to lectin or antigen. J. Immunol. 133:3054-3061.

35. Narumiya, S., M. Hirata, T. Nanba, T. Nikaido, Y. Taniguchi, Y. Tagaya, M. Okada, H. Mitsuya H., and J. Yodoi. 1987. Activation of interleukin-2 receptor gene by forskolin and cyclic AMP analogues. Biochem. Biophys. Res. Commun. 143:753-760.

36. Lucey, D. R., A. Nicholson-Weller, and P. F. Weller. 1989. Mature human eosinophils have the capacity to express HLA-DR. Proc. Natl. Acad. Sci. USA. 86:1348-1351.

37. Yagita, H., M. Nakata, A. Azuma, T. Nitta, T. Takeshita, K. Sugamura, and $\mathrm{K}$. Okumura. 1989. Activation of peripheral blood T cells via the $\mathrm{p} 75$ interleukin 2 receptor. J. Exp. Med. 170:1445-1450.

38. Nagler, A., L. L. Lanier, and J. H. Phillips. 1990. Constitutive expression of high affinity interleukin 2 receptors on human CD16 natural killer cells in vivo. J. Exp. Med. 171:1527-1533.

39. Aribia, M. B., N. Moire, D. Metivier, C. Vaquero, O. Lantz, D. Olive, B. Carpenter, and A. Senik. 1989. IL-2 receptors on circulating natural killer cells and T lymphocytes. J. Immunol. 142:490-499.

40. Birchenall-Sparks, M. C., W. L. Farrar, D. Rennick, P. L. Kilian, and F. W. Ruscetti. 1986. Regulation of expression of the interleukin-2 receptor on hematopoietic cells by interleukin-3. Science (Wash. DC) 233:456-458.

41. Cruikshank, W. W., J. S. Berman, A. C. Theodore, J. Bernardo, and D. M. Center. 1987. Lymphokine activation of T4+ lymphocytes and monocytes. $J$. Immunol. 138:3817-3823.

42. Smith, K. A., and D. A. Cantrell. 1985. Interleukin 2 regulates its own receptors. Proc. Natl. Acad. Sci. USA. 82:864-868.

43. Yoshimoto, T., K. Nakanishi, K. Matsui, S. Hirose, K. Hiroishi, T. Tanaka, T. Hada, T. Hamaoka, and K. Higashino. 1990. IL-5 up-regulates but IL-4 down-regulates IL-2R expression on a cloned B lymphoma line. J. Immunol. 144:183-190.

44. Valerius, T., R. Repp, J. R. Kalden, and E. Platzer. 1990. Effects of interferons on human eosinophils in comparison with other cytokines. J. Im munol. 145:2950-2958.

45. Prin, L., J. C. Ameisen, J. Plumas, V. Gruart, S. Loiseau, O. Bletry, P. Fenaux, M. Capron, and A. Capron. 1990. High levels of soluble interleukin-2 receptor in the serum of 30 patients with idiopathic hypereosinophilic syndrome. Br. J. Haematol. 74:233-237.

46. Assawi, M., B. Bradley, P. K. Jeffrey, A. J. Frew, A. J. Wardlaw, G. Knowles, B. Assoufi, J. V. Collins, S. Durham, and A. B. Kay. 1990. Identification of activated $\mathrm{T}$ lymphocytes and eosinophils in bronchial biopsies in stable atopic asthma. Am. Rev. Respir. Dis. 142:1407-1413.

47. Lotze, M. T., M. C. Custer, and S. A. Rosenberg. 1986. Intraperitoneal administration of interleukin-2 in patients with cancer. Arch. Surg. 121:13731379.

48. Huland, E., and H. Huland. 1989. Local continuous high dose interleukin 2: a new therapeutic model for the treatment of advanced bladder carcinoma. Cancer Res. 49:5469-5474

49. Nakamura, Y., T. Ozaki, H. Yanagawa, S. Yasuoka, and T. Ogura. 1990. Eosinophil colony-stimulating factor induced by administration of interleukin-2 into the pleural cavity of patients with malignant pleurisy. Am. J. Respir. Cell. Mol. Biol. 3:291-300.

50. Silberstein, D. S., D. D. Schoof, M. L. Rodrick, P. Tai, C. J. F. Spry, J. R David, and T. J. Eberlein. 1989. Activation of eosinophils in cancer patients treated with IL-2 and IL-2-generated lymphokine-activated killer cells. J. Immunol. 142:2162-2167.

51. Enokihara, H., S. Furusawa, H. Nakakubo, H. Kajitani, S. Nagashima, K. Saito, H. Shishido, Y. Hitoshi, K. Takatsu, T. Noma, A. Shimizu, and T. Honjo. 1989. T cells from eosinophilic patients produce interleukin- 5 with interleukin-2 stimulation. Blood 73:1809-1813.

52. Secor, W. E., S. J. Stewart, and D. G. Colley. 1990. Eosinophils and immune mechanisms. VI. The synergistic combination of granulocyte-macrophage colony-stimulating factor and IL-5 accounts for eosinophil-stimulation promoter activity in Schistosoma mansoni-infected mice. J. Immunol. 144:1484-1489.

53. Thorne, K. J. I., B. A. Richardson, J. Taverne, D. J. Williamson, M. A Vadas, and A. E. Butterworth. 1986. A comparison of eosinophil-activating factor with other monokines and lymphokines. Eur. J. Immunol. 16:1143-1149.

54. Fujisawa, T., R. Abu-Ghazaleh, H. Kita, C. J. Sanderson, and G. J. Gleich. 1990. Regulatory effect of cytokines on eosinophil degranulation. J. Immunol. 144:642-646. 